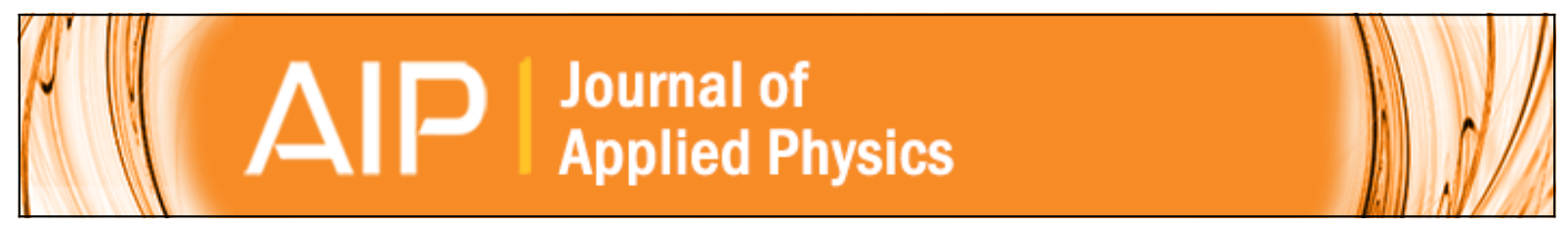

\title{
A phenomenological fitting curve for the magnetocaloric effect of materials with a second-order phase transition
}

Q. Y. Dong, H. W. Zhang, J. R. Sun, B. G. Shen, and V. Franco

Citation: Journal of Applied Physics 103, 116101 (2008); doi: 10.1063/1.2913166

View online: http://dx.doi.org/10.1063/1.2913166

View Table of Contents: http://scitation.aip.org/content/aip/journal/jap/103/11?ver=pdfcov

Published by the AIP Publishing

\section{Articles you may be interested in}

Procedure for numerical integration of the magnetocaloric effect

J. Appl. Phys. 112, 063920 (2012); 10.1063/1.4754561

Magnetocaloric materials with first-order phase transition: thermal and magnetic hysteresis in LaFe11.8Si1.2 and $\mathrm{Ni2} .21 \mathrm{Mn} 0.77 \mathrm{Ga} 1.02$ (invited)

J. Appl. Phys. 111, 07 A910 (2012); 10.1063/1.3670987

Magnetocaloric effect and its implementation in critical behavior study of Mn4FeGe3- x Si x intermetallic compounds

J. Appl. Phys. 110, 113915 (2011); 10.1063/1.3669390

The magnetocaloric effect in soft magnetic amorphous alloys

J. Appl. Phys. 101, 09 C503 (2007); 10.1063/1.2709409

Influence of mixing the low-valent transition metal atoms ( $Y, Y^{*}=\mathrm{Cr}, \mathrm{Mn}, \mathrm{Fe}$ ) on the properties of the quaternary Co $2\left[\mathrm{Y} 1-\mathrm{x} Y \mathrm{x}^{*}\right] \mathrm{Z}(\mathrm{Z}=\mathrm{Al}, \mathrm{Ga}, \mathrm{Si}, \mathrm{Ge}$, or $\mathrm{Sn})$ Heusler compounds

J. Appl. Phys. 101, 073910 (2007); 10.1063/1.2714502

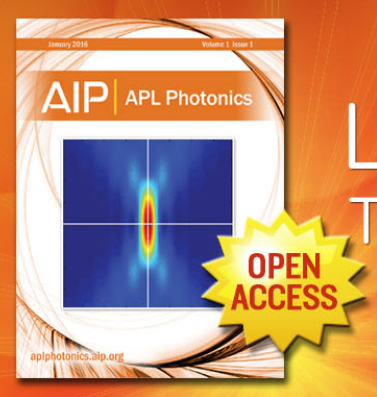

Launching in 2016!

The future of applied photonics research is here

A $\mid \begin{aligned} & \text { APL } \\ & \text { Photonics }\end{aligned}$ 


\title{
A phenomenological fitting curve for the magnetocaloric effect of materials with a second-order phase transition
}

\author{
Q. Y. Dong, ${ }^{1}$ H. W. Zhang, ${ }^{1, a)}$ J. R. Sun, ${ }^{1}$ B. G. Shen, ${ }^{1}$ and V. Franco ${ }^{2}$ \\ ${ }^{1}$ State Key Laboratory for Magnetism, Institute of Physics and Center for Condensed Matter Physics, \\ Chinese Academy of Sciences, Beijing 100080, People's Republic of China \\ ${ }^{2}$ Departamento Física de la Materia Condensada, ICMSE-CSIC, Universidad de Sevilla, P.O. Box 1065, \\ 41080 Sevilla, Spain
}

(Received 22 November 2007; accepted 3 March 2008; published online 2 June 2008)

\begin{abstract}
The magnetic entropy change of polycrystalline samples $\mathrm{Gd}, \mathrm{La}\left(\mathrm{Fe}_{0.92} \mathrm{Co}_{0.08}\right)_{11.83} \mathrm{Al}_{1.17}$, $\mathrm{LaFe}_{10.8} \mathrm{Si}_{2.2}, \mathrm{Mn}_{5} \mathrm{Ge}_{2.7} \mathrm{Ga}_{0.3}, \mathrm{Nd}_{2} \mathrm{AlFe}_{13} \mathrm{Mn}_{3}$, and $\mathrm{TbCo}_{2}$ with a second-order phase transition has been investigated. A uniform phenomenological function that describes the magnetic entropy change is found for these materials. This could be of great benefit for the design of magnetic refrigerators. The field dependence of the critical exponent for the variation in the maximum entropy change with field is studied. The critical exponent value of $2 / 3$, which is predicted by the mean field theory, is only satisfied for moderate field values. The refrigerant capacity is analyzed and compared to the predictions of the fitting function. @ 2008 American Institute of Physics. [DOI: 10.1063/1.2913166]
\end{abstract}

Since the discovery of the giant magnetocaloric effect (MCE) in $\mathrm{Gd}_{5} \mathrm{Si}_{2} \mathrm{Ge}_{2},{ }^{1}$ much interest has been focused on the magnetic refrigeration near room temperature due to its numerous potential advantages over vapor-compression refrigeration. ${ }^{2-4}$ The isothermal magnetic entropy change $\left(\Delta S_{M}\right)$ is an important parameter for evaluating the refrigerant properties. ${ }^{5}$ Currently, large $\Delta S_{M}$ has been found in materials with a magnetostructural first-order phase transition. However, some problems such as hysteresis and time dependence should be overcome before using those materials as magnetic refrigerants. In fact, the cooling power of a magnetic refrigerator is a product of the operation frequency and the relative cooling power of the refrigerant. Therefore, although materials with a second-order phase transition may have a lower peak entropy change, this can be compensated by their faster response, which can facilitate the increase in the operation frequency in refrigerator appliances. ${ }^{6}$

Recently, the investigation of the magnetic field $(H)$ dependence of $\Delta S_{M}$ has been renewed in materials with a second-order phase transition. The critical exponent $n$ for the variation in the maximum $\Delta S_{M}\left(\Delta S_{M}^{\mathrm{pk}}\right)$ with $H$ has been studied in detail. ${ }^{7-12}$ Furthermore, a universal behavior is fulfilled for $\Delta S_{M}$ curves measured for various field changes in amorphous alloys. ${ }^{9-11}$ Later on, it was evidenced for lanthanidebased materials such as $\mathrm{Gd}$ and $\left(\mathrm{Er}_{1-x} \mathrm{Dy}_{x}\right) \mathrm{Al}_{2}{ }^{12}$ However, up until now, no exact analytical form of this universal curve has been given because in a general case, the $\Delta S_{M}(H, T)$ curve involves the hypergeometric function, where $T$ is the temperature. In this work, a uniform phenomenological function is found to describe the variation in $\Delta S_{M}$ with $H$ and $T$ for some different samples. As a consequence, the $\Delta S_{M}(H, T)$ curve for any of these materials can be determined by knowing its Curie temperature $\left(T_{C}\right)$, peak entropy change $\Delta S_{M}^{p k}$, and two additional reference temperatures. Meanwhile, the

\footnotetext{
a) Author to whom correspondence should be addressed. Electronic mail: hwzhang@aphy.iphy.ac.cn.
}

variation in the critical exponent $n$ with field has been obtained, which is necessary to obtain $\Delta S_{M}^{\mathrm{pk}}(H)$. Although it is usually assumed that the value of $n$ corresponds to the mean field approach $\left(\Delta S_{M}^{\mathrm{pk}} \sim H^{2 / 3}\right)$, using the actual value for each particular sample can solve the negative intercept problem in fitting the experimental data of these materials in Ref. 8. The clarification of this problem can provide directions for the design of a magnetic refrigerant.

The preparation, x-ray diffraction analyses, and magnetic measurements of the polycrystalline samples $\mathrm{Gd}$, $\mathrm{La}\left(\mathrm{Fe}_{0.92} \mathrm{Co}_{0.08}\right)_{11.83} \mathrm{Al}_{1.17}, \quad \mathrm{LaFe}_{10.8} \mathrm{Si}_{2.2}, \quad \mathrm{Mn}_{5} \mathrm{Ge}_{2.7} \mathrm{Ga}_{0.3}$, $\mathrm{Nd}_{2} \mathrm{AlFe}_{13} \mathrm{Mn}_{3}$, and $\mathrm{TbCo}_{2}$ are described elsewhere. ${ }^{8}$ The value of $\Delta S_{M}$ was calculated from magnetization data by using the integrated Maxwell relations.

The phenomenological universal curve can be constructed by ${ }^{9}(1)$ normalizing all the $\Delta S_{M}(T)$ curves by using their respective maximum value $\Delta S_{M}^{\mathrm{pk}}$, namely, $\Delta S^{\prime}$ $=\Delta S_{M}(T) / \Delta S_{M}^{\mathrm{pk}}$, and (2) rescaling the temperature axis below and above $T_{C}$, as defined in Eq. (1) with an imposed constraint that the position of two additional reference points in the curve corresponds to $\theta= \pm 1$,

$$
\theta= \begin{cases}-\left(T-T_{C}\right) /\left(T_{r 1}-T_{C}\right), & T \leq T_{C}, \\ \left(T-T_{C}\right) /\left(T_{r 2}-T_{C}\right), & T>T_{C},\end{cases}
$$

where $T_{r 1}$ and $T_{r 2}$ are the temperature of the two reference points that, for the present study, have been selected as those corresponding to $\Delta S_{M}\left(T_{r 1,2}\right)=1 / 2 \Delta S_{M}^{\mathrm{pk}}$. Figure 1 shows the $\theta$ dependence of $\Delta S^{\prime}$ for typical field changes for all of the studied samples. One can clearly find that all the experimental points distribute on one universal curve. The universal curve can be well fitted by a Lorentz function,

$$
\Delta S^{\prime}=\frac{a}{b+(\theta-c)^{2}},
$$

where $a, b$, and $c$ are the free parameters. By taking into account the asymmetry of the curve, two different set of constants have to be used: for $T \leq T_{C}, a=1.69 \pm 0.06, b$ 


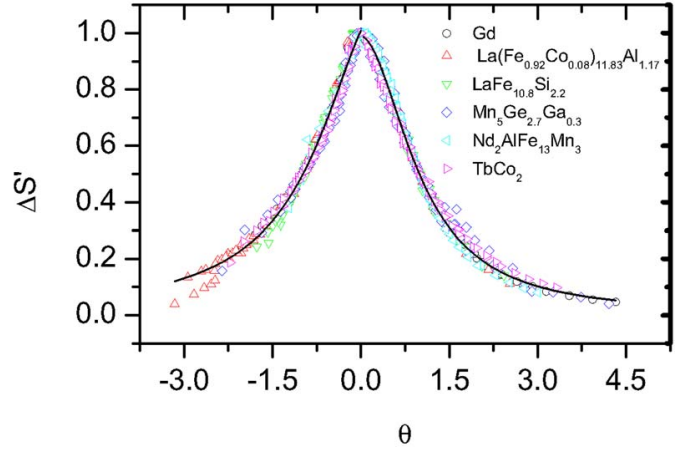

FIG. 1. (Color online) $\theta$ dependence of $\Delta S^{\prime}$ for the studied samples for typical field changes $(0-1,2,3,4$, and $5 \mathrm{~T})$; the solid line is the fit to Eq. (2).

$=1.54 \pm 0.03, c=0.38 \pm 0.03$; and for $T>T_{C}, a=1.05 \pm 0.02$, $b=1.06 \pm 0.02, c=-0.03 \pm 0.01$. In the present cases, $a, b$, and $c$ are common for these materials. Recently, it was demonstrated that a single reference temperature should be enough for constructing the universal curve. ${ }^{13,14}$ Although this alternative approach works well for most of the present materials, the data for $\mathrm{La}\left(\mathrm{Fe}_{0.92} \mathrm{Co}_{0.08}\right)_{11.83} \mathrm{Al}_{1.17}$ and $\mathrm{LaFe}_{10.8} \mathrm{Si}_{2.2}$ alloys fail to collapse on one universal curve when only a single reference temperature is used. This peculiarity is being currently investigated in more detail. ${ }^{15}$

According to Eq. (2), only the position and magnitude of the peak, namely, $\left(T_{C}, \Delta S_{M}^{\mathrm{pk}}\right)$, and two reference temperatures $T_{r 1}$ and $T_{r 2}$, are needed to characterize the entropy change, where $T_{r 1}<T_{C}$ and $T_{r 2}>T_{C}$. That is to say, to translate $\Delta S^{\prime}(\theta)$ into the "real" $\Delta S_{M}(T)$, you need only these values that are determined by the properties of the materials. Thus, incomplete $\Delta S_{M}(T)$ curves, which are experimentally determined from a small temperature span in the vicinity of $T_{C}$ for the isothermal magnetization measurements, can be easily transformed into the complete curves, which is a helpful tool for the evaluation of material properties such as the refrigerant capacity (RC). Furthermore, engineers can use this function to analyze the influence of material parameters on the design of a magnetic refrigerator. Certainly, the extension of Eq. (2) obtained from the present materials to other compositions needs to be verified experimentally and theoretically in the future.

Among the three points mentioned above, the point $\left(T_{C}, \Delta S_{M}^{\mathrm{pk}}\right)$ is the most important. So, we check the field dependence of $\Delta S_{M}^{\mathrm{pk}}$ in detail. First, to determine the field dependence of the experimental $\left|\Delta S_{M}\right|$ of the studied materials, a local exponent ${ }^{16}$ is calculated as

$$
n=\frac{d \ln \left|\Delta S_{M}\right|}{d \ln H} .
$$

The value of $n$ depends on the values of field and temperature. On the basis of a mean field approach, the field dependence of the magnetic entropy change at $T_{C}, \Delta S_{M}^{\mathrm{pk}}$, has been predicted by $n=2 / 3 .^{7-9}$ In a general case, $n$ is related to the critical exponents of the material. ${ }^{9}$ Figure 2 shows the $\theta$ dependence of the local exponent $n$ for the studied samples for typical field changes (not larger than $5 \mathrm{~T}$ ). At $T_{C}$, an increase in $n$ is observed with the decrease in the field change. This can be associated to the nonsaturation of the

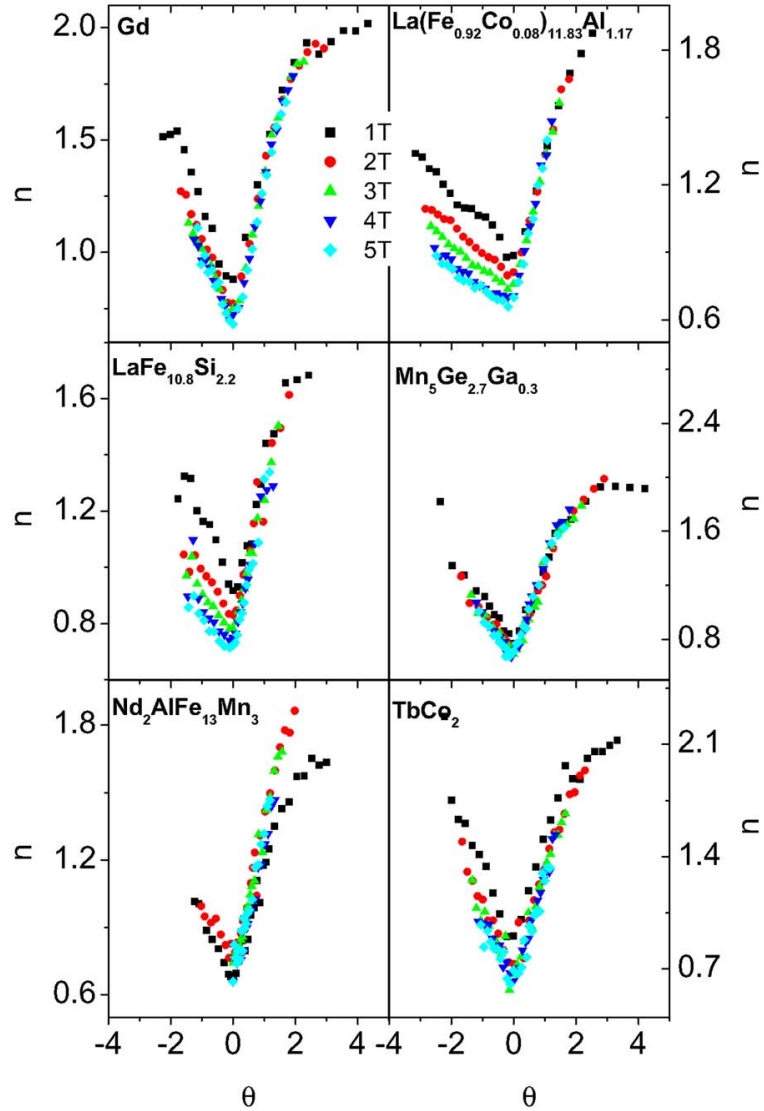

FIG. 2. (Color online) $\theta$ dependence of the local exponent $n$ for the studied samples for typical field changes.

sample. Thus, due to the local exponent $n$ being larger than $2 / 3$ for small field changes, the negative intercept can be eliminated in fitting the relationship of $\left|\Delta S_{M}\right|$ and $H^{2 / 3}$ at $T_{C}$ in Ref. 8. Apart from the lowest field data, all of the $n(\theta)$ curves in Fig. 2 collapse, within the experimental error, onto a single one. There are two remarkable exceptions: $\mathrm{La}\left(\mathrm{Fe}_{0.92} \mathrm{Co}_{0.08}\right)_{11.83} \mathrm{Al}_{1.17}$ and $\mathrm{LaFe}_{10.8} \mathrm{Si}_{2.2}$ compounds, wherein a continuous decrease in $n$ with increasing field is found below $T_{C}$. This can be related to the previously mentioned failure in obtaining a universal curve for these two alloys when a single reference temperature is used. ${ }^{15}$ As an example, Fig. 3 shows the field change dependence of $n$ in the vicinity of $T_{C}(=241 \mathrm{~K})$ for $\mathrm{LaFe}_{10.8} \mathrm{Si}_{2.2}$ compound. As

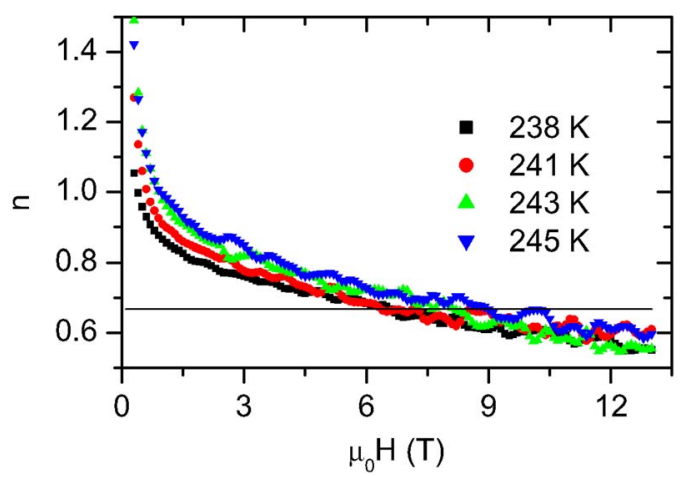

FIG. 3. (Color online) Field change dependence of $n$ in the vicinity of $T_{C}$ for $\mathrm{LaFe}_{10.8} \mathrm{Si}_{2.2}$ compound; the solid line is the $n=2 / 3$ reference. 


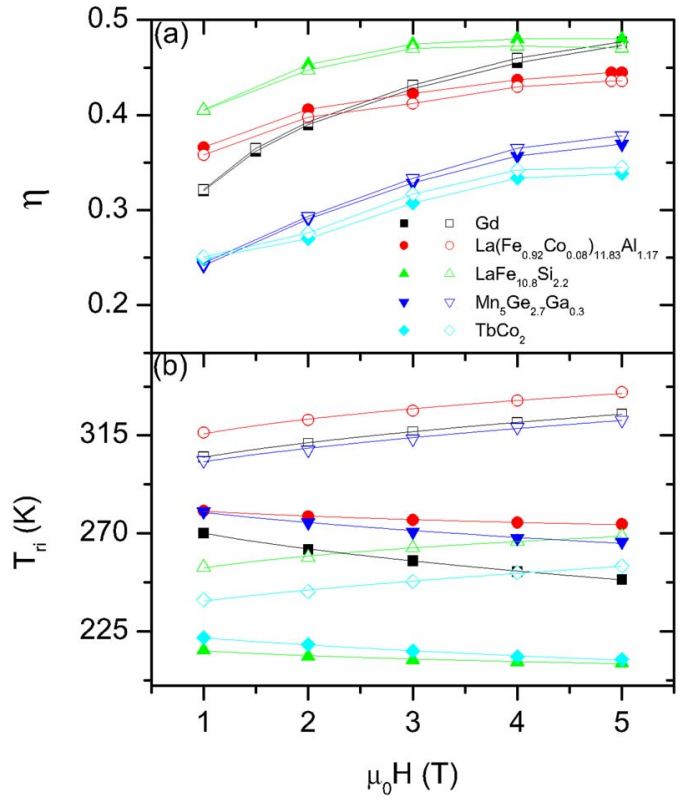

FIG. 4. (Color online) Field change dependence of (a) $\eta$ [the closed symbols are for experimental data and the open symbols for the results of Eq. (2); the lines are a guide for the eye $]$ and (b) $T_{r i}(i=1,2)$ (the closed symbols are for $T_{r 1}$ and the open symbols are for $T_{r 2}$; the lines are a fit to a power law of the field).

shown in Fig. 3, $n$ initially decreases with increasing field changes. Then, it slowly changes with further increasing field changes, but it is not saturating even for a field change of $0-13 \mathrm{~T}$ at $T_{C}$.

Besides the $\Delta S_{M}^{\mathrm{pk}}$, the $\mathrm{RC}$ is another important parameter in characterizing the MCE. The numerical integration of the area under the $\left|\Delta S_{M}\right|$ curve between $T_{r 1}$ and $T_{r 2}$ is used as

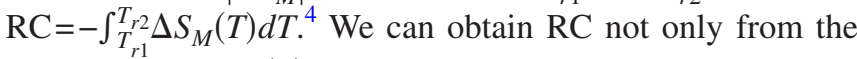
experimental $\Delta S_{M}(T)$ curves but also from the curves given by Eq. (2). The former is labeled as $\mathrm{RC}_{\text {expt }}$, and the latter as $\mathrm{RC}_{\text {calc }}$. Now, we define the utilization ratio of a given magnetic refrigerant as $\eta=\mathrm{RC} / A$, where $A$ is the RC between 0 $\mathrm{K}$ and infinite. According to the Maxwell relations, $A$ equals to $-\int_{0}^{\infty} \Delta S_{M}(T) d T=\Delta H M_{s},{ }^{17}$ where $M_{s}$ is the saturation magnetization at $0 \mathrm{~K}$. Similarly, we have $\eta_{\text {expt }}=\mathrm{RC}_{\text {expt }} / A$ and $\eta_{\text {calc }}=\mathrm{RC}_{\text {calc }} / A$. Figure $4(\mathrm{a})$ shows the field change dependence of $\eta$ for the present materials. The value of $M_{s}$ for the present materials refers to Ref. 8. As shown in Fig. 4(a), $\eta_{\text {calc }}$ is consistent with $\eta_{\text {expt }}$, which further confirms the validity of Eq. (2). Both $\eta_{\text {calc }}$ and $\eta_{\text {expt }}$ increase with increasing field and reach about 0.48 for a field change of $5 \mathrm{~T}$ for $\mathrm{Gd}$ and $\mathrm{LaFe}_{10.8} \mathrm{Si}_{2.2}$. Figure 4(b) shows the variation in $T_{r i}(i=1,2)$ with the magnetic field change, which can guide the design of a magnetic refrigerant. In Fig. 4(b), the lines correspond to the fitting of the reference temperature data to a power law of the field, as was recently predicted. ${ }^{13,14}$ For a low field change $(0-1.5 \mathrm{~T})$, which can be supplied by a $\mathrm{NdFeB}$ per- manent magnet, the value of $\Delta T\left(=T_{r 2}-T_{r 1}\right)$ is larger than 40 $\mathrm{K}$ for $\mathrm{Gd}, \mathrm{La}\left(\mathrm{Fe}_{0.92} \mathrm{Co}_{0.08}\right)_{11.83} \mathrm{Al}_{1.17}$, and $\mathrm{LaFe}_{10.8} \mathrm{Si}_{2.2}$. This is also beneficial for future applications in magnetic refrigeration.

In summary, the magnetic entropy change can be characterized by one universal curve for all of the studied materials. The universal curve can be fitted by a Lorentz function, $\Delta S^{\prime}=a /\left[b+(\theta-c)^{2}\right]$, where $a=1.69 \pm 0.06, b=1.54 \pm 0.03$, and $c=0.38 \pm 0.03$ for $T \leq T_{C}$; and $a=1.05 \pm 0.02, b$ $=1.06 \pm 0.02$, and $c=-0.03 \pm 0.01$ for $T>T_{C}$. Based on this function, only the coordinates of the peak $\left(T_{C}, \Delta S_{M}^{\mathrm{pk}}\right)$ and the two reference temperatures $T_{r 1}$ and $T_{r 2}$ are needed to describe the whole entropy change curves. The exponent $n$ initially rapidly decreases with increasing field changes and then slowly for $0-13 \quad \mathrm{~T}$ field change for $\mathrm{La}\left(\mathrm{Fe}_{0.92} \mathrm{Co}_{0.08}\right)_{11.83} \mathrm{Al}_{1.17}$ and $\mathrm{LaFe}_{10.8} \mathrm{Si}_{2.2}$ compounds. In $0-5 \mathrm{~T}$, the value of the utilization ratio $\eta$ reaches about 0.48 for $\mathrm{Gd}$ and $\mathrm{LaFe}_{10.8} \mathrm{Si}_{2.2}$, being smaller for the other studied compositions.

This work was supported by the State Key Project of Fundamental Research and the National Natural Science Foundation of China (Grant No. 10774178). V.F. acknowledges the financial support of the Spanish Government and EU FEDER (Project MAT 2007-65227).

${ }^{1}$ V. K. Pecharsky and K. A. Gschneidner, Jr., Phys. Rev. Lett. 78, 4494 (1997).

${ }^{2}$ Z. B. Guo, Y. W. Du, J. S. Zhu, H. Huang, W. P. Ding, and D. Feng, Phys. Rev. Lett. 78, 1142 (1997)

${ }^{3}$ O. Tegus, E. Bruck, K. H. J. Buschow, and F. R. de Boer, Nature (London) 415, 150 (2002).

${ }^{4}$ K. A. Gschneidner, Jr., V. K. Pecharsky, and A. O. Tsokol, Rep. Prog. Phys. 68, 1479 (2005).

${ }^{5}$ N. A. de Oliveira, P. J. von Ranke, M. V. Tovar Costa, and A. Troper, Phys. Rev. B 66, 094402 (2002).

${ }^{6}$ M. D. Kuz'min, Appl. Phys. Lett. 90, 251916 (2007).

${ }^{7}$ H. Oesterreicher and F. T. Parker, J. Appl. Phys. 55, 4334 (1984).

${ }^{8}$ Q. Y. Dong, H. W. Zhang, J. L. Shen, J. R. Sun, and B. G. Shen, J. Magn. Magn. Mater. 319, 56 (2007).

${ }^{9}$ V. Franco, J. S. Blázquez, and A. Conde, Appl. Phys. Lett. 89, 222512 (2006).

${ }^{10}$ V. Franco, C. F. Conde, and A. Conde, Appl. Phys. Lett. 90, 052509 (2007).

${ }^{11}$ V. Franco, J. S. Blázquez, M. Millán, J. M. Borrego, C. F. Conde, and A. Conde, J. Appl. Phys. 101, 09C503 (2007).

${ }^{12}$ V. Franco, A. Conde, V. K. Pecharsky, and K. A. Gschneidner, Jr., Europhys. Lett. 79, 47009 (2007).

${ }^{13}$ V. Franco, J. S. Blázquez, and A. Conde, J. Appl. Phys. 103, 07B316 (2008).

${ }^{14}$ V. Franco, A. Conde, J. M. Romero-Enrique, and J. S. Blázquez (unpublished).

${ }^{15}$ V. Franco, A. Conde, Q. Y. Dong, and H. W. Zhang (unpublished).

${ }^{16}$ T. D. Shen, R. B. Schwarz, J. Y. Coulter, and J. D. Thompson, J. Appl. Phys. 91, 5240 (2002).

${ }^{17}$ A. M. Tishin and Y. I. Spichkin, The Magnetocaloric Effect and Its Applications, 1st ed. (Instutute of Physics, Bristol, Philadelphia, 2003), Vol. 1, Chap. 11, p. 351. 